\title{
CRESCIMENTO DE MUDAS DE MARACUJAZEIRO EM SUBSTRATO ALTERNATIVO COM FERTILIZANTE DE LIBERAÇÃO CONTROLADA
}

\author{
Rosiney França Mendes ${ }^{1}$, Jamayra Conceição de Araújo ${ }^{2}$, Romeu de Carvalho Andrade Neto ${ }^{3}$, \\ James Maciel de Araújo ${ }^{1}$, João Paulo Maia Guilherme ${ }^{4}$
}

\begin{abstract}
RESUMO - Na região norte, principalmente, no Acre, Amazonas e Pará são produzidos diversos resíduos provenientes do processamento de frutas nativas que, na maioria dos locais de beneficiamento, são descartados sem nenhum aproveitamento. Portanto, buscar alternativas de aproveitamento, sobretudo, como substrato para produção de mudas é de grande valia para a sustentabilidade do sistema produtivo. Objetivouse, neste trabalho, avaliar o crescimento de mudas de maracujazeiro em substrato alternativo com adubo de liberação controlada. O experimento foi conduzido na casa de vegetação da Embrapa Acre. O delineamento experimental utilizado foi o inteiramente casualizado, em esquema fatorial $5 \times 2$, sendo 5 formulações de substrato e 2 doses de adubo (com e sem), com 4 repetições. Os tratamentos foram constituídos da casca de cupuaçu triturada e substrato comercial, puros (100\%), e misturados nas proporções $25 \%, 50 \%$ e $75 \%$ v/v. Ambos, com e sem o adubo de liberação controlada. Foram avaliadas as variáveis respostas: Porcentagem de germinação, índice de velocidade de emergência, altura de planta, diâmetro do caule, número de folha, massas secas; da parte área, das raízes, total, e relação raiz/parte aérea. Nos tratamentos contendo a mistura dos substratos, bem como, no substrato comercial puro, houve influência significativa $(p<0,05)$ para todas as variáveis analisadas, as quais foram destacadas com a presença do adubo de liberação controlada. O substrato alternativo formado a partir da mistura da casca de cupuaçu triturada com o MECPLANT Florestal nas proporções 25\%, $50 \%$ e $75 \%$, acrescido do fertilizante de liberação controlada, proporciona elevado crescimento das mudas de maracujazeiro quando comparado sem a adição do fertilizante.
\end{abstract}

Palavras chave: nutrição de plantas, Passiflora edulis, resíduo vegetal.

\section{GROWTH OF PASSION FRUIT SEEDLINGS IN ALTERNATIVE SUBSTRATE WITH CONTROLLED RELEASE FERTILIZER}

\begin{abstract}
In the northern region, mainly in Acre, Amazonas and Pará are produced several residues from the processing of native fruits that, in most processing sites, are discarded without any harnessing. Therefore, seeking alternatives of utilization, about everything as a substrate for seedling production, is of great value for the sustainability of the production system. This work aimed evaluate the growth of passion fruit seedlings in alternative substrate with controlled release fertilizer. The experiment was conducted in the greenhouse of the Brazilian Agricultural Research Corporation in Rio Branco Acre. The was used completely randomized design was completely randomized in a $5 \times 2$ factorial scheme, with 5 substrate formulations and 2 fertilizer doses (with and without), with 4 replications. The treatments consisted of crushed
\end{abstract}

\footnotetext{
${ }^{1}$ Engenheiro Agrônomo, Doutorando no Programa de Pós-Graduação em Produção Vegetal da Universidade Federal do Acre, Rodovia BR-364, Km 04, Rio Branco, Acre, CEP: 69920-900. E-mail para correspondência: rosiney.agro@gmail.com

2 Engenheira Florestal, Doutoranda no Programa de Pós-Graduação em Produção Vegetal da Universidade Federal do Acre, Rodovia BR-364, Km 04, Rio Branco, Acre, CEP: 69920-900.

3 Engenheiro Agrônomo Dr., Pesquisador da Empresa Brasileira de Pesquisa Agropecuária/Acre, Rodovia BR-364, Km 14, Rio Branco, Acre, CEP: 69900-970.

${ }^{4}$ Engenheiro Agrônomo Ms., do Instituto de Meio Ambiente do Acre, Rua Rui Barbosa 135, Centro, Rio Branco, Acre, CEP: 69900-120.
} 
cupuassu bark and commercial substrate, pure (100\%), and mixed in 25\%, 50\% and $75 \%$ v/v proportions. Both with and without controlled release fertilizer. The following variables were evaluated: germination percentage, emergence speed index, plant height, stem diameter, leaf number, dry mass: of the aerial part, of root, total, and root/ aerial part ratio. In the treatments containing the mixture of substrates, as well as in the pure commercial substrate, there was a significant influence $(p<0.05)$ for all analyzed variables, which were highlighted with the presence of the controlled release fertilizer. The alternative substrate formed from the mixture of the crushed cupuassu bark with MECHPLANT Forestry in proportions 25\%, 50\% and 75\%, plus controlled release fertilizer, provides high growth of passion fruit seedlings, when compared without the addition of the fertilizer.

Keywords: mineral nutrition of plant, Passiflora edulis, plant residue.

\section{INTRODUÇÃO}

O maracujazeiro amarelo (Passiflora edulis L.) é uma frutífera que se desenvolve bem em região de clima tropical. Oferece rápido retorno econômico e oportunidade de receita distribuída na maior parte do ano (Pires et al., 2008; Barros et al., 2013). O Brasil é o maior produtor mundial de maracujá amarelo (Filho et al., 2010; Souto et al., 2017). Com aproximadamente 41 mil hectares cultivados e produtividade média de 13,5 t.ha ${ }^{-1}$ (IBGE, 2017).

O processo de produção de mudas deve ter como finalidade a obtenção de plantas vigorosas para formação ou renovação de pomares, dessa maneira, é fundamental a escolha de bons substratos para obtenção de mudas de qualidade (Guerra et al., 2017).

Bons substratos devem apresentar propriedades físico-químicas adequadas, fornecendo água, oxigênio e os nutrientes necessários para o bom desenvolvimento inicial da planta. Alguns substratos apesar de apresentarem boas características físicas, são pobres em nutrientes, devido a isso, é necessário a sua suplementação com adubo de qualidade, em doses adequadas e preferencialmente com mecanismos de liberação controlada, afim de evitar perdas (Elli et al., 2013).

Segundo Mendonça et al. (2008) os fertilizantes de liberação controlada são alternativas viáveis, pois permitem que os nutrientes sejam disponibilizados de maneira contínua à planta, minimizando os riscos de deficiências. Dinalli et al. (2012) relatam que para maior eficácia do substrato em relação à nutrição das mudas durante a fase inicial, tem-se utilizado como alternativa os fertilizantes de liberação controlada, devido sua eficiência na liberação contínua de nutrientes, redução de perdas ocasionadas pela lixiviação, proporcionando nutrição constante à planta durante todo período de crescimento.

$\mathrm{Na}$ região norte, principalmente, no Acre, Amazonas e Pará são produzidos diversos resíduos provenientes do processamento de frutas nativas que, na maioria dos locais de beneficiamento, são descartados sem nenhum aproveitamento. Portanto, buscar alternativas de uso, sobretudo, como substrato para produção de mudas é de grande valia para a sustentabilidade do sistema produtivo.

Pelo exposto, objetivou-se, neste trabalho, avaliar o crescimento de mudas de maracujazeiro em substrato alternativo com fertilizante de liberação controlada.

\section{MATERIAL E MÉTODOS}

O experimento foi conduzido na casa de vegetação da Empresa Brasileira de Pesquisa Agropecuária (EMBRAPA), localizada na BR-364, Km 14 em Rio Branco Acre.

O delineamento experimental utilizado foi $\mathrm{o}$ inteiramente casualizado, em esquema fatorial $5 \times 2$, sendo 5 formulações de substrato e 2 doses de adubo (com e sem), com 4 repetições. As unidades experimentais estiveram constituídas por recipientes plástico transparente com volume de $250 \mathrm{~mL}$, com cinco pequenos orifícios na base para drenagem da água proveniente da irrigação. Os tratamentos foram constituídos de: Substrato comercial puro $=\mathrm{T} 1$; Casca de cupuaçu triturada pura $=\mathrm{T} 2 ; 50 \%$ Casca de cupuaçu triturada e 50\% substrato comercial $=\mathrm{T} 3 ; 75 \%$ substrato comercial e $25 \%$ casca de cupuaçu triturada $=\mathrm{T} 4 ; 75 \%$ casca de cupuaçu triturada e $25 \%$ substrato comercial $=\mathrm{T} 5$. Ambos com e sem o fertilizante de liberação controlada.

A casca do fruto de cupuaçu foi obtida no projeto RECA, a qual passou por um processo de desidratação a pleno sol (em temperatura média de $25{ }^{\circ} \mathrm{C}$ ) por 90 dias, e depois, fragmentada manualmente com auxílio de um pilão. Posteriormente, foi triturada em moinho mecânico. O substrato comercial utilizado foi o MECPLANT Florestal, formulado a partir da casca de pinus bioestabilizada, vermiculita, corretivo de acidez e macronutrientes. Ambos os substratos, foram passados em peneira com malha de $10 \mathrm{~mm}$ para a separação das frações mais grosseiras. Os atributos químicos e físicos 
dos materiais utilizados na formulação dos substratos estão especificados na Tabela 1. Os tratamentos contendo os dois substratos nas proporções estudadas e puros com a adição do fertilizante de liberação controlada, foram homogeneizados em betoneira por 10 minutos, e depois distribuídos nos recipientes (unidades experimentais).

Tabela 1 - Atributos químicos e físicos dos materiais utilizados na formulação dos substratos. Rio Branco, Acre, 2019

\begin{tabular}{|c|c|c|c|c|c|c|c|}
\hline \multirow{2}{*}{ Substrato } & \multirow{2}{*}{$\mathrm{pH}$} & \multicolumn{6}{|c|}{ Macronutrientes (g/kg) } \\
\hline & & $\mathrm{N}$ & $\mathrm{P}$ & $\mathrm{K}$ & $\mathrm{Ca}$ & $\mathrm{Mg}$ & $\mathrm{S}$ \\
\hline Comercial & 4,9 & 9,8 & 0,3 & 1,8 & 7,1 & 1,4 & 1,0 \\
\hline Cupuaçu & 7,4 & 6,6 & 2,1 & 1,4 & 12 & 4,3 & 0,9 \\
\hline \multirow{2}{*}{ Substrato } & \multicolumn{7}{|c|}{ Micronutrientes $(\mathrm{mg} / \mathrm{kg})$} \\
\hline & $\mathrm{B}$ & \multicolumn{2}{|c|}{$\mathrm{Cu}$} & $\mathrm{Fe}$ & \multicolumn{2}{|c|}{$\mathrm{Mn}$} & $\mathrm{Zn}$ \\
\hline Comercial & 9,9 & \multicolumn{2}{|c|}{14,4} & 2900 & \multicolumn{2}{|c|}{207,8} & 28,8 \\
\hline Cupuaçu & 23,4 & \multicolumn{2}{|c|}{35,3} & 6200 & \multicolumn{2}{|c|}{199,1} & 72,1 \\
\hline \multirow{3}{*}{ Substrato } & \multicolumn{7}{|c|}{ Atributos físicos e relação carbono/nitrogênio } \\
\hline & \multirow{2}{*}{$\begin{array}{c}\mathrm{CO} \\
(\mathrm{g} / \mathrm{kg}) \\
\end{array}$} & \multirow{2}{*}{$\begin{array}{c}\text { Relação } \\
\mathrm{C} / \mathrm{N}\end{array}$} & \multirow{2}{*}{$\begin{array}{c}\mathrm{CE} \\
\left(\mathrm{dS} . \mathrm{m}^{-1}\right)\end{array}$} & \multicolumn{2}{|c|}{$\mathrm{DU}$} & DS & CRA \\
\hline & & & & \multicolumn{3}{|c|}{-------(kg/m³)------- } & $\% \mathrm{v} / \mathrm{v}$ \\
\hline Comercial & 339,5 & 34,64 & 0,9 & \multicolumn{2}{|c|}{500,0} & 200,0 & 62,3 \\
\hline Cupuaçu & 432,9 & 65,59 & 1,1 & \multicolumn{2}{|c|}{488,6} & 460,5 & 83,5 \\
\hline
\end{tabular}

$\mathrm{CO}$ = Carbono orgânico; $\mathrm{C} / \mathrm{N}$ = Relação carbono/nitrogênio; $\mathrm{CE}$ = Condutividade elétrica; $\mathrm{DU}=$ Densidade úmida; $\mathrm{DS}=$ Densidade seca; $\mathrm{CRA}$ = Conteúdo relativo de água.

Nos tratamentos contendo o fertilizante de liberação controlada, foi adicionado $8 \mathrm{~g} / \mathrm{L}$ do Basacote ${ }^{\circledR}$ Mini 3M, o qual é composto dos macronutrientes (N-PK-Mg-S:13-6-16-1,4-10 em \%, respectivamente) e dos micronutrientes (Cu-Fe-Mn-Mo:0,05-0,26-0,06-0,015 em $\%$, respectivamente). Com período de liberação de três meses.

As plantas foram propagadas a partir de sementes, as quais foram extraídas de frutos maduros da cultivar BRS Gigante Amarelo, e preparadas para a semeadura conforme Alexandre et al. (2004). A irrigação nas unidades experimentais foi realizada por meio de microaspersão intermitente, mantendo a umidade em $70 \%$. Os cuidados fitossanitários foram realizados diariamente por meio de vistorias preventivas quanto ao ataque de pragas ou o surgimento de doenças. Durante toda a condução do experimento não foi necessário nenhum tipo de controle, tendo em vista que não houve a ocorrência de pragas e/ou doenças.

Aos 12 dias após a semeadura foram avaliadas as variáveis; porcentagem de germinação (PG) e índice de velocidade de emergência (IVE), conforme Maguire (1962), e aos 45 dias após a emergência; altura da planta (AP), diâmetro do caule (DC), número de folha por planta (NFP), massas secas da parte aérea (MSPA), das raízes (MSR), total (MST) e relação raiz/parte aérea (RRPA), seguindo a metodologia de Costa et al. (2015).
Os dados obtidos foram submetidos aos testes de Shapiro e Wilk (1965) para verificação da normalidade dos erros, e Cochran (1941) para verificação da homogeneidade das variâncias, e quando atendidos os referidos pressupostos, foi realizada à análise de variância, a qual sendo significativa $(\mathrm{p}<0,05)$, aplicou-se o teste de Tukey (1949) ao nível 5\% de probabilidade para comparação das médias. Como ferramenta auxiliar para a execução das análises, utilizou-se o software estático R (versão 3.6.1).

\section{RESULTADOS E DISCUSSÃO}

A porcentagem de germinação e o índice de velocidade de emergência foram influenciados $(\mathrm{P}<0,05)$ pelos tratamentos estudados (Figuras 1 e 2). No substrato comercial puro (T1) e nos provenientes da mistura com a casca de cupuaçu triturada (T3, T4 e T5), independentemente da presença ou ausência do fertilizante de liberação controlada, verificou-se, alto desempenho e similaridade dos resultados entre os tratamentos. Contrariamente, no substrato casca de cupuaçu triturada pura (T2), mesmo com a presença do fertilizante de liberação controlada, verificou-se redução de $70 \%$ das referidas variáveis, quando comparada aos demais tratamentos.

A baixa porcentagem germinativa no substrato casca de cupuaçu triturada pura, está relacionado com o fato 
de que no mesmo, verificou-se baixa umidade, provocada pela formação de selamento na camada superficial, dificultando desta forma, a infiltração e consequentemente absorção de água pelas sementes. Segundo Carvalho \& Nakagawa (2012), as sementes necessitam de um mínimo de água para atingirem o nível de hidratação adequado, e assim, se inicie o processo germinativo, e se não ocorrer essa condição, o processo germinativo é comprometido. Consequentemente, segundo os referidos autores, isso influencia de forma direta nos baixos índices de velocidade de emergência, pois a mesma é dependente da germinação. Fato que corrobora com os resultados observados neste estudo.

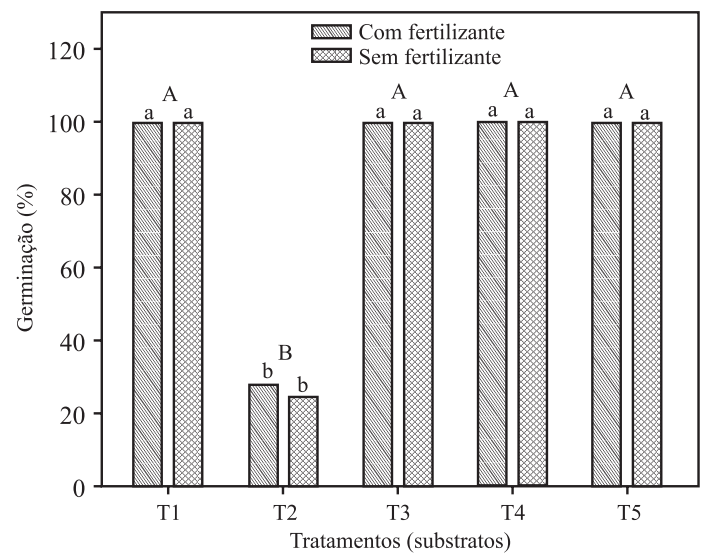

Figura 1 - Porcentagem de germinação aos 12 após a semeadura. Colunas seguidas da mesma letra minúscula dentro de cada tratamento e maiúscula entre os tratamentos, não diferem $(\mathrm{P}>0,05)$ entre si pelo teste de Tukey.

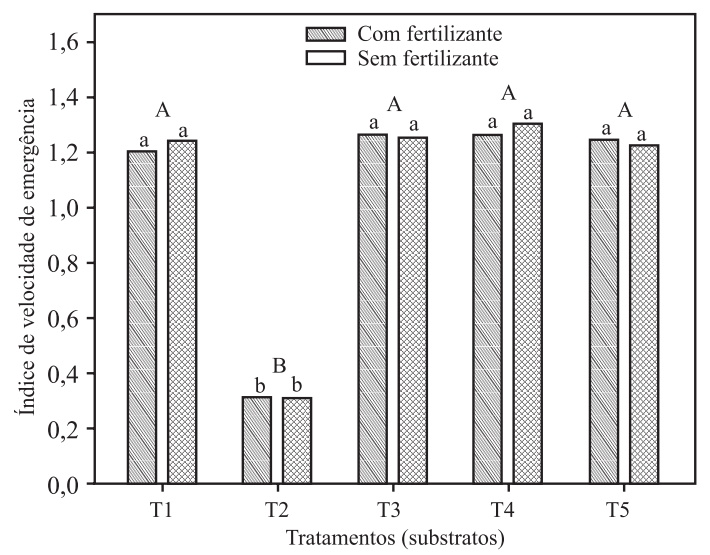

Figura 2 - Índice de velocidade de emergência. Colunas seguidas da mesma letra minúscula dentro de cada tratamento e maiúscula entre os tratamentos, não diferem $(\mathrm{P}>0,05)$ entre si pelo teste de Tukey.
No tratamento contendo apenas a casca de cupuaçu triturada pura, mesmo com o fertilizante de liberação controlada (T2), as plântulas de maracujazeiro senesceram após a emergência, exatamente por isso, não constam resultados dos referidos tratamentos nas Tabelas 2 e 3 .

O número de folha por planta foi influenciado $(\mathrm{P}<0,05)$ pelos tratamentos estudados (Tabela 2). No substrato comercial (T1), bem como os provenientes da mistura com a casca de cupuaçu triturada (T3, T4 e T5) com o fertilizante de liberação controlada, promoveram maior número de folhas nas mudas de maracujazeiro. Conforme Scalon et al. (2003) o número de folhas é um importante indicador de bom desempenho das mudas, pois está relacionado com a área foliar e pode ser considerado como um importante parâmetro para produtividade, dada a importância nos processos fotossintéticos e consequentemente na produção. Sousa et al. (2011) mencionaram que o número e o tempo de permanência das folhas em plena atividade na planta podem resultar no aumento da taxa de interceptação de radiação solar, com consequente aumento no metabolismo e na produtividade das mesmas.

A altura de planta e o diâmetro do caule, a exemplo do número de folha, foram influenciados $(\mathrm{P}<0,05)$ pelos tratamentos analisados (Tabela 2). Os tratamentos com substrato comercial (T1) e os provenientes de sua mistura com a casca de cupuaçu triturada (T3, T4 e T5), ambos com o fertilizante de liberação controlada, promoveram os maiores crescimento em altura e diâmetro do caule das mudas de maracujazeiro.

O maior crescimento em altura e diâmetro do caule das mudas de maracujazeiro nos tratamentos com o fertilizante de liberação controlada, está relacionado com o fornecimento de nutrientes em proporções adequadas a cada etapa de crescimento, visto que a absorção não é constante ao longo do ciclo (Marana et al., 2008).

As massas secas da parte aérea, das raízes, total e relação raiz/parte aérea foram influenciadas $(\mathrm{P}<0,05)$ pelos tratamentos analisados (Tabela 3). As mudas de maracujazeiro acumularam mais massa seca nos tratamentos com o fertilizante de liberação controlada. Contrariamente, as maiores relação raiz/parte aérea, ocorreram nos tratamentos sem o fertilizante.

Kato et al. (2018) também constataram incremento de massa seca total em função da aplicação do fertilizante de liberação controlada em mudas de maracujazeiro amarelo. Conforme Cavatte et al. (2011) a formação de biomassa pelo vegetal pode ser conceituada por uma relação fisiológica simples, a qual se baseia na radiação absorvida e na eficácia 
de conversão em matéria seca, sendo esse processo afetado por algum estresse abiótico. Neste contexto, as mudas de maracujazeiro cultivadas nos substratos sem o fertilizante de liberação controlada podem terem sofrido estresse por déficit nutricional razão pela qual pode ter comprometido a atividade fotossintética, que nestas condições é reduzida e/ou paralisada pela falta de nutrientes, sobretudo, por nitrogênio (Malavolta, 2006).

Tabela 2 - Número de folhas por planta (NFP), altura da planta (AP) e diâmetro do caule (DC) das mudas de maracujazeiro cv. BRS Gigante Amarelo, aos 45 dias após a emergência. Rio Branco, Acre, 2019.

\begin{tabular}{|c|c|c|c|c|c|c|}
\hline \multirow[t]{2}{*}{ Tratamentos } & \multicolumn{2}{|c|}{ NFP } & \multicolumn{2}{|c|}{$\begin{array}{c}\text { AP } \\
(\mathrm{cm})\end{array}$} & \multicolumn{2}{|c|}{$\begin{array}{c}\mathrm{DC} \\
(\mathrm{mm})\end{array}$} \\
\hline & $\mathrm{Cf}$ & Sf & $\mathrm{Cf}$ & $\mathrm{Sf}$ & $\mathrm{Cf}$ & Sf \\
\hline $\mathrm{T} 1$ & $9,73 \mathrm{aA}$ & $6,25 \mathrm{aB}$ & $21,75 \mathrm{bA}$ & $8,85 \mathrm{aB}$ & $3,21 \mathrm{aA}$ & $2,19 \mathrm{aB}$ \\
\hline $\mathrm{T} 3$ & $10,00 \mathrm{aA}$ & $5,25 \mathrm{aB}$ & $28,75 \mathrm{abA}$ & $5,67 \mathrm{abB}$ & $3,32 \mathrm{aA}$ & $1,57 \mathrm{bB}$ \\
\hline $\mathrm{T} 4$ & $10,77 \mathrm{aA}$ & $6,13 \mathrm{aB}$ & 30,66 aA & $8,25 \mathrm{aB}$ & $3,20 \mathrm{aA}$ & $2,20 \mathrm{aA}$ \\
\hline T5 & $9,85 \mathrm{aA}$ & $2,75 \mathrm{bB}$ & $26,02 \mathrm{abA}$ & $2,45 \mathrm{bB}$ & $3,13 \mathrm{aA}$ & $1,19 \mathrm{cB}$ \\
\hline
\end{tabular}

$\mathrm{Cf}=$ Com fertilizante; $\mathrm{Sf}=$ Sem fertilizante. Médias seguidas de mesma letra minúscula na coluna, e maiúscula na linha, não diferem $(\mathrm{P}>0,05)$ entre si pelo teste de Tukey.

Tabela 3 - Massas secas da parte aérea (MSPA), das raízes (MSR), total (MST) e relação raiz/parte aérea das mudas de maracujazeiro cv. BRS Gigante Amarelo, aos 45 dias após a emergência. Rio Branco, Acre, 2019

\begin{tabular}{|c|c|c|c|c|c|c|c|c|}
\hline \multirow[t]{2}{*}{ Tratamentos } & \multicolumn{2}{|c|}{$\begin{array}{c}\text { MSPA }^{1} \\
(\mathrm{mg})\end{array}$} & \multicolumn{2}{|c|}{$\begin{array}{l}\mathrm{MSR}^{1} \\
(\mathrm{mg})\end{array}$} & \multicolumn{2}{|c|}{$\begin{array}{l}\mathrm{MST}^{1} \\
(\mathrm{mg})\end{array}$} & \multicolumn{2}{|c|}{$\begin{array}{c}\text { RRPA }^{1} \\
(\mathrm{mg})\end{array}$} \\
\hline & $\mathrm{Cf}$ & Sf & $\mathrm{Cf}$ & $\mathrm{Sf}$ & $\mathrm{Cf}$ & $\mathrm{Sf}$ & $\mathrm{Cf}$ & Sf \\
\hline $\mathrm{T} 1$ & $1423 \mathrm{aA}$ & $418 \mathrm{aB}$ & $373 \mathrm{aA}$ & $203 \mathrm{aB}$ & $1795 \mathrm{aA}$ & $620 \mathrm{aB}$ & $0,26 \mathrm{aB}$ & $0,49 \mathrm{bA}$ \\
\hline $\mathrm{T} 3$ & $2325 \mathrm{aA}$ & $190 \mathrm{aB}$ & $513 \mathrm{aA}$ & $103 \mathrm{bB}$ & $2838 \mathrm{aA}$ & $293 \mathrm{bB}$ & $0,22 \mathrm{aB}$ & $0,54 \mathrm{bA}$ \\
\hline $\mathrm{T} 4$ & $465 \mathrm{aA}$ & $225 \mathrm{aB}$ & $590 \mathrm{aA}$ & $193 \mathrm{aB}$ & $2840 \mathrm{aA}$ & $658 \mathrm{aB}$ & $0,26 \mathrm{aB}$ & $0,41 \mathrm{bA}$ \\
\hline $\mathrm{T} 5$ & $2028 \mathrm{aA}$ & $33 \mathrm{bB}$ & $513 \mathrm{aA}$ & $29 \mathrm{cB}$ & $2540 \mathrm{aA}$ & $62 \mathrm{cB}$ & $0,25 \mathrm{aB}$ & $0,89 \mathrm{aA}$ \\
\hline CV (\%) & \multicolumn{2}{|c|}{6,58} & \multicolumn{2}{|c|}{4,92} & \multicolumn{2}{|c|}{4,67} & \multicolumn{2}{|c|}{10,50} \\
\hline
\end{tabular}

${ }^{1}$ Dasdos transformados por $\ln (\mathrm{x}) . \mathrm{Cf}=\mathrm{Com}$ fertilizante; $\mathrm{Sf}=\mathrm{Sem}$ fertilizante. Médias seguidas de mesma letra minúscula na coluna, e maiúscula na linha, não diferem $(\mathrm{P}>0,05)$ entre si pelo teste de Tukey.

Para os resultados de relação raiz/parte aérea, constatou-se que as mudas de maracujazeiro apresentaram maior desenvolvimento radicular quando foram cultivadas nos substratos sem o fertilizante de liberação controlada. Segundo Zhu et al. (2010) isso ocorre por se tratar de um importante processo adaptativo das plantas, no qual a manutenção do crescimento das raízes durante períodos demenor disponibilidade de água e nutrientes, permite a exploração de maiores áreas, e consequentemente, o maior acúmulo de biomassa das raízes, em comparação com a parte aérea.
Outro fator relevante que pode ter contribuído para o aumento da relação raiz/parte aérea nos tratamentos sem o fertilizante de liberação controlada é que a demanda nutricional das mudas de maracujazeiro pode não terem sido atendidas, principalmente em nitrogênio. Situação que pode ter sido agravada em virtude da alta relação $\mathrm{C} / \mathrm{N}=65,59$ (Tabela 1 ) da casca de cupuaçu triturada. Nesta situação o nitrogênio foi mobilizado, ocasionando assim deficiência deste nutriente às plantas (Kiehl, 2004). Consequentemente, isso provocou alterações na distribuição de fotoassimilados 
entre as raízes e a parte aérea, tendo como consequência o aumento na relação raízes/parte aérea, que é o efeito mais característico (Rufty et al., 1990).

\section{CONCLUSÃO}

O substrato alternativo formado a partir da mistura da casca de cupuaçu triturada com o MECPLANT Florestal nas proporções $25 \%, 50 \%$ e $75 \%$, acrescido do fertilizante de liberação controlada, proporciona elevado crescimento das mudas de maracujazeiro quando comparado sem a adição do fertilizante.

\section{AGRADECIMENTOS}

Ao projeto RECA pelo fornecimento dos resíduos agroindustriais. À EMBRAPA/AC e UFAC pelo apoio à pesquisa, e ao CNPq e CAPES pela concessão das bolsas de mestrado, doutorado e produtividade (processo 313552/2017-7).

\section{LITERATURA CITADA}

ALEXANDRE, R.S.; JÚNIOR,A. W.; SILVANEGREIROS, J. R.; PARIZZOTTO, A.; BRUCKNER, C. H. Germinação de sementes de genótipos de maracujazeiro. Pesquisa Agropecuária Brasileira, Brasília, DF, v. 39, n. 12, p. 12391245, 2004.

BARROS, C. M. B.; MÜLLER, M. M. L.; BOTELHO, R. V.; MICHALOVICZ, L.; VICENSI, M.; NASCIMENTO, R. Substratos com compostos de adubos verdes e biofertilizante via foliar na formação de mudas de maracujazeiro-amarelo. Magistra, Cruz das Almas, v. 26, n. 4, p. 482-492, 2013.

CARVALHO, N. M.; NAKAGAWA, J. Sementes: Ciência, tecnologia e produção. 5.ed. FUNEP, Jaboticabal, Brasil, 2012. 590p.

CAVATTE, P. C.; MARTINS, S. C. V.; MORAIS, L. E.; SILVA, P. E. M.; SOUZA, L. T. A fisiologia dos estresses abióticos. Melhoramento de plantas para condições de estresses abióticos. Suprema, Visconde do Rio Branco, p. 39-79, 2011.

COCHRAN, W. G. The distribution of the largest of a set of estimated variances as a fraction of their total. Annals of Eugenics, Medford, v. 22, n. 11, p. 47-52, 1941.

COSTA, E.; SANTOS, L. C. R.; CARVALHO, C.; LEAL, P. A. M.; AMARAL, G. V. Volumes de substratos comerciais, solo e composto orgânico afetando a formação de mudas de maracujazeiro-amarelo em diferentes ambientes de cultivo. Revista Ceres, Viçosa, MG, v. 58, n. 2, p. 216-222, 2015.

DINALLI, R. P.; CASTILHO, R. M. M.; GAZOLA, R. N. Utilização de adubos de liberação controlada na produção de mudas de Vigna radiata L. Revista Científica Eletrônica de Agronomia, Garça, v. 21, n.1, p. 10-15, 2012.

ELli, E. F.; CANTARELli, E. B.; CARON, B. O.; MONTEIRO, G. C.; PAVAN, M. A.; PEDRASSANI, M.; ELOY, E. Osmocote $\AA$ no desenvolvimento e comportamento fisiológico de mudas de pitangueira. Comunicata Scientiae, Bom Jesus, PI, v. 4, n. 4, p. 377 , 2013.

FILHO, G. C. N.; RONCATTO, G.; RUGGIERO, C.; OLIVEIRA, J. C.; MALHEIROS, E. B. estudo da enxertia hipocotiledonar do maracujazeiro-amarelo sobre dois portaenxertos, através de microscopia eletrônica de varredura. Revista Brasileira de Fruticultura, Jaboticabal, v. 32, n. 2, p. 647-652, 2010.

GUERRA, M. S.; BARBOSA, M. S.; COSTA, E.; VIEIRA, G. H. C. Recipiente biodegradável e substratos para mudas de maracujazeiro. Revista de Agricultura Neotropical, Cassilândia, v. 4, n. 3, p. 50-54, 2017.

INSTITUTO BRASILEIRO DE GEOGRAFIA E ESTATÍSTICA. Produção Agrícola Municipal. Disponível em: https://sidra.ibge.gov.br/tabela/5457\#resultado. Acesso em: 15 junho de 2019.

KATO, D. S.; SILVA, C. M.; HIGUCHI, M. T.; BAUCHROWITZ, I. M.; SANTOS NETO, J.; SHIMIZU, G. D.; OLIVEIRA, A. F. Produção de mudas de maracujá amarelo submetidas a doses crescentes de adubação de liberação controlada. Revista Terra \& Cultura, Londrina, v. 34, n. especial, p. 310-320, 2018.

KIEHL, E. J. Manual de compostagem: Maturação e qualidade do composto. 4. ed. Piracicaba: E. J. Kiehl, 2004. $173 p$.

MAGUIRE, J. D. Speed of germination aid in selection and evaluation for emergence and vigour. Crop Science, Madison, v. 2, n. 2, p. 176-177, 1962.

MALAVOLTA, E. Manual de nutrição mineral de plantas. São Paulo, Ceres, 2006. 638 p.

MARANA, J. P.; MIGLIORANZA, E.; PÁDUA FONSECA, E.; HIROSHI KAINUMA, R. Índices de qualidade e crescimento de mudas de café produzidas em tubetes. Ciência Rural, Santa Maria, RS, v. 38, n. 1, p. 3945, 2008. 
MENDONÇA, V.; ARRUDA, A. N.; ABREU, H. A. S.; TEIXEIRA, G. A.; HAFLE, O. M. Diferentes ambientes e Osmocote ${ }^{\circledR}$ na produção de mudas de tamarindeiro (Tamarindus indica). Ciência e Agrotecnologia, Lavras, v.32, n.2, p.391-397, 2008

MENDONÇA, V.; TOSTA, M. S.; MACHADO, J. R.; GOULART JUNIOR, S. A. R.; TOSTA, J. S.; BISCARO, G. A. Fertilizante de liberação controlada na formação de mudas de maracujazeiro amarelo. Ciência e Agrotecnologia, Lavras, v. 31, n. 2, p. 344-348, 2007.

PIRES, A. A.; MONNERAT, P. H.; MARCIANO, C. R.; PINHO, L. G. R.; ZAMPIROLLI, P. D.; ROSA, R. C. C.; MUNIZ, R. A. Efeito da adubação alternativa do maracujazeiro-amarelo nas características químicas e físicas do solo. Revista Brasileira de Ciência do Solo, Viçosa, MG, v. 32, n. 5, p. 1997-2005, 2008.

RUFTY, T. W.; MACKOWN, C. T.; VOLK, R. J. Alteration in nitrogen assimilation and partitioning in nitrogen stressed plants. Physiologia Plantarum, v. 79, n. 1, p. 85- 95, 1990.

SCALON, Q. S. D. P.; MUSSURY, M. R.; RIGONI, R. M.; FILHO, S. H. Crescimento inicial de mudas de Bombacopsis glabra (Pasq.) A. Robyns sob condição de sombreamento. Revista Árvore, Viçosa, MG, v. 27, n. 6, p. 753-758, 2003.

SHAPIRO, S. S.; WILK, M. B. An analysis of variance test for normality (complete samples). Biometrika, Oxford, v. 52 , n. $3 / 4$, p. $591-611,1965$

SOUSA, A. G. O.; FERNANDES, D. C.; ALVES, A. M.; FREITAS, J. B.; NAVES, M. M. V. Nutritional quality and protein value of exotic almonds and nut from the Brazilian Savanna compared to peanut. Food Research International, Barking, v. 44, n. 7, p. 2319 -2325, 2011.

SOUTO, A. G. L.; CREMASCO, J. P. G.; MAITAN, M. Q.; AZEVEDO, J. L. F.; RIBEIRO, M. R.; SANTOS, C. E. M. Seed germination and vigor of passion fruit hybrids. Comunicata Scientiae, Bom Jesus, PI, v. 8, n. 1, p. 134-138, 2017.

TUKEY, J. W. Comparing individual means in the analysis of variance. Biometrics, Alexandria, v. 5, n. 2, p. 99-114, 1949.

ZHU, J.; BROWN, K. M.; LYNCH, J. P. Root cortical aerenchyma improves the drought tolerance of maize (Zea mays L.). Plant, cell and Environment, v. 33. n. 5, p. 740749, 2010.

Recebido para publicação em 20/08/2019 e aprovado em 04/12/2019. 